\title{
Hereditary Brachydactyly Associated with Hypertension
}

\author{
N. BILGINTURAN, S. ZILELI, S. KARACADAG, and T. PIRNAR
}

Departments of Paediatrics, Internal Medicine, and Radiology, School of Medicine, Hacettepe University, Turkey

Summary. A family showing brachydactyly associated with hypertension is reported. The number and location of the involved bones are quite different from the other cases with brachydactyly reported in the literature. Additionally, all our patients have high blood pressure. We believe that the disorder reported here represents a hitherto undescribed entity. It is thought that both hypertension and brachydactyly are transmitted as an autosomal dominant trait by a single pleiotropic gene or by two closely located genes.

Hereditary brachydactylies are well-known clinical entities since Farabee's original paper (1903). The same author described an autosomal inheritance pattern for the trait (1905). Later, much has been written on the inheritance and morphology of brachydactyly and associated anomalies. The paper written by Bell (1951) is particularly important for the classification of the condition. On the basis of Bell's criteria, McKusick (1971) has recently reviewed these entities, with some addition of the new types of brachydactylies.

One type is characterized by short metacarpals and metatarsals. There is wide variability in the number of digits affected. The patients are moderately short of stature and have round faces but do not have ectopic calcification, mental retardation, or cataract as in pseudo-pseudohypoparathyroidism which is an otherwise similar entity.

In the family reported here we present in addition clinical, radiological, and genetic findings of brachydactyly, short stature, and hypertension.

\section{Material and Method}

The index case, a 30-year-old female, was discovered when she brought her son to Hacettepe Children's Hospital for the evaluation of his congenital heart disease. The family history revealed many members with hypertension and brachydactyly. She further stated that a significant number of family members had been lost at a relatively young age as a result of cerebrovascular accident. The pedigree (Fig. 1), indicates that those who

Received 9 February 1973. have brachydactyly, either by examination or by history, also have or have had hypertension and were commonly lost due to cerebrovascular accident. The family lives on the eastern Black Sea coast of Turkey. We visited that area and saw 73 members of the family. Height, weight, and blood pressure were obtained for each individual, in addition to routine physical examination (Table I); dermatoglyphic analyses were also done. Five of the 21 affected individuals were hospitalized for further studies including hypertensive tests. The following studies were carried out in every patient: blood urea nitrogen determination, creatinine

TABLE I

AGE SEX, HEIGHT, AND BLOOD PRESSURE MEASUREMENTS OF BRACHYDACTYLOUS PATIENTS

\begin{tabular}{l|c|c|c|c}
\hline \multicolumn{1}{c|}{ Case } & Age & Sex & Height $(\mathrm{cm})$ & Blood Pressure (mm/Hg) \\
\hline III.6 & 65 & F & 141 & $200 / 155$ \\
\hline IV.3 & 40 & F & 143 & $190 / 110$ \\
IV.6 & 33 & M & 155 & $240 / 170$ \\
IV.12 & 36 & M & 151 & $230 / 155$ \\
IV.14 & 34 & M & 152 & $195 / 110$ \\
IV.16 & 28 & M & 151 & $165 / 110$ \\
IV.18 & 46 & F & 147 & $190 / 140$ \\
IV.19 & 42 & M & 154 & $270 / 160$ \\
IV.22 & 36 & M & 154 & $195 / 125$ \\
IV.26 & 30 & F & 145 & $180 / 115$ \\
IV.27 & 21 & M & 156 & $160 / 105$ \\
\hline V.1 & 17 & F & 141 & $145 / 110$ \\
V.4 & $2 \frac{1}{2}$ & F & 81 & $110 / 65$ \\
V.10 & 10 & F & 112 & $135 / 75$ \\
V.12 & 6 & M & 102 & $110 / 70$ \\
V.13 & 3 & F & 86 & $105 / 70$ \\
V.14 & 1 & F & 68 & $100 / 60$ \\
V.17 & 16 & F & 135 & $155 / 90$ \\
V.19 & 8 & M & 106 & $135 / 85$ \\
V.20 & 7 & F & 105 & $140 / 95$ \\
& 6 & F & 103 & $130 / 80$ \\
\hline
\end{tabular}




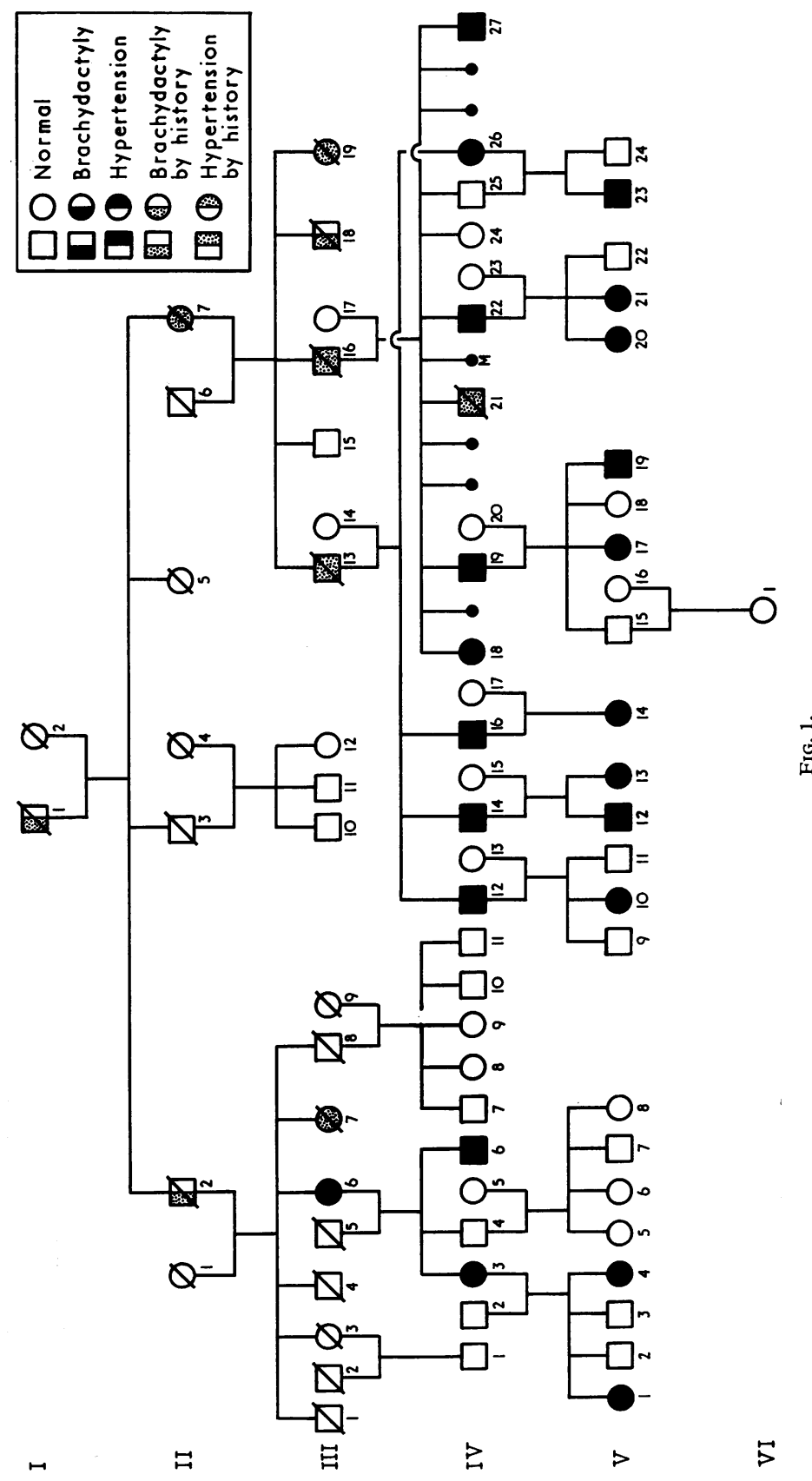



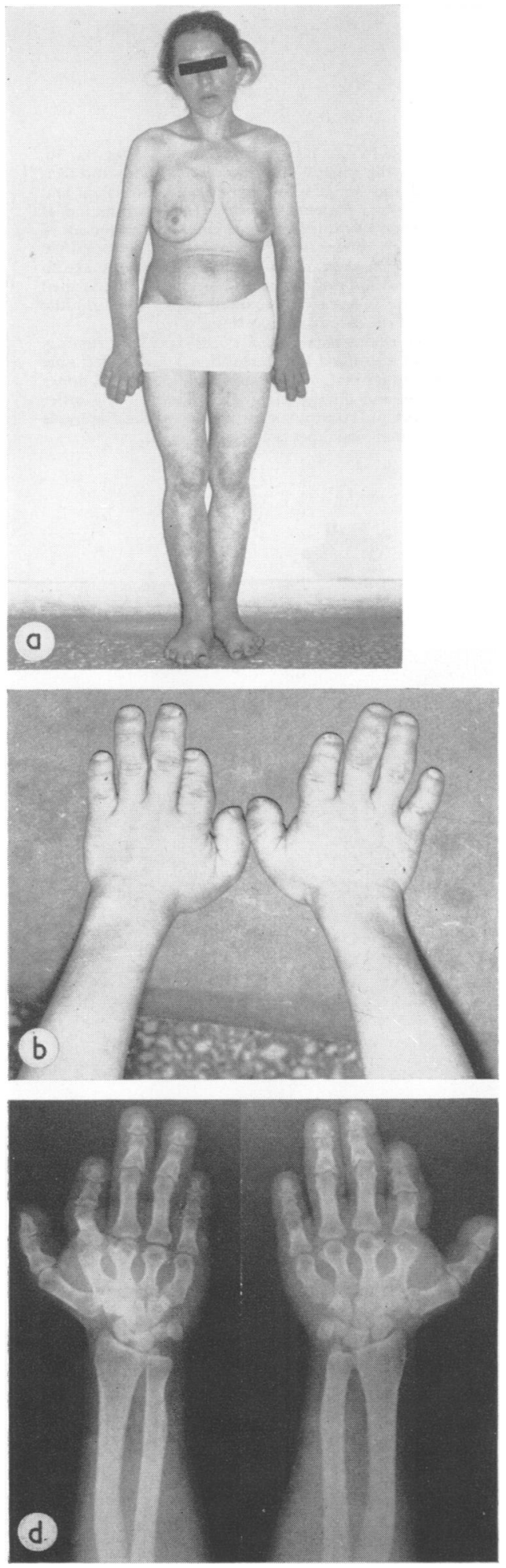

Fig. 2. The index case. Figs. 2a, 2b, and $2 c$ show the general appearance and hands and feet. Figs. $2 \mathrm{~d}$ and $2 \mathrm{e}$ show $x$-rays of the hands and feet. Radiological examination of the hands shows symmetrical and marked shortening of all metacarpal bones with the exception of the first metacarpal on the left side. A similar shortening is present also in all the phalanges of the fingers with the exception of the proximal phalanges of the third and fourth fingers on both sides; these phalanges appear relatively normal. Because of the shortening, the involved metacarpals and phalanges are relatively broad and stubby. There is also irregularity of the articular surfaces in the proximal interphalangeal joints of the second, third and fourth fingers where the proximal epiphyses of the middle phalanges are cone-shaped.

The first and fourth metatarsals on the right side and the first, fourth and fifth metatarsals on the left side are shortened. There is similar shortening of al! the phalanges of the toes, with narrowing of the diaphyses of the proximal phalanges producing an hour-glass appearance. Fusion between the middle and distal phalanges of the fifth toes bilaterally is observed.
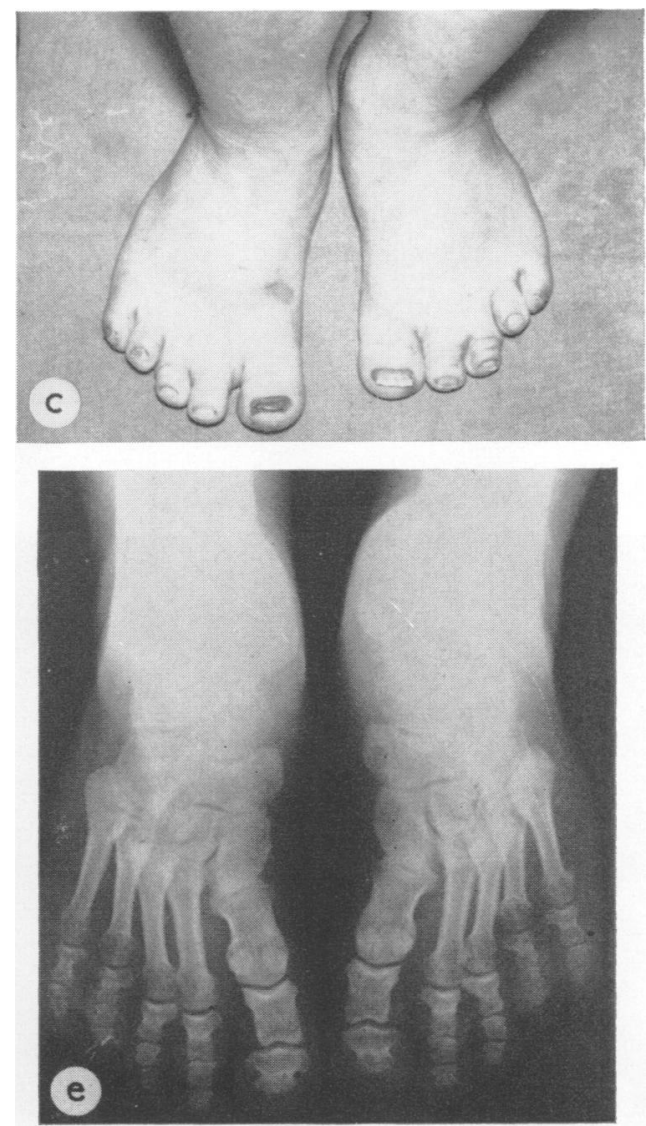

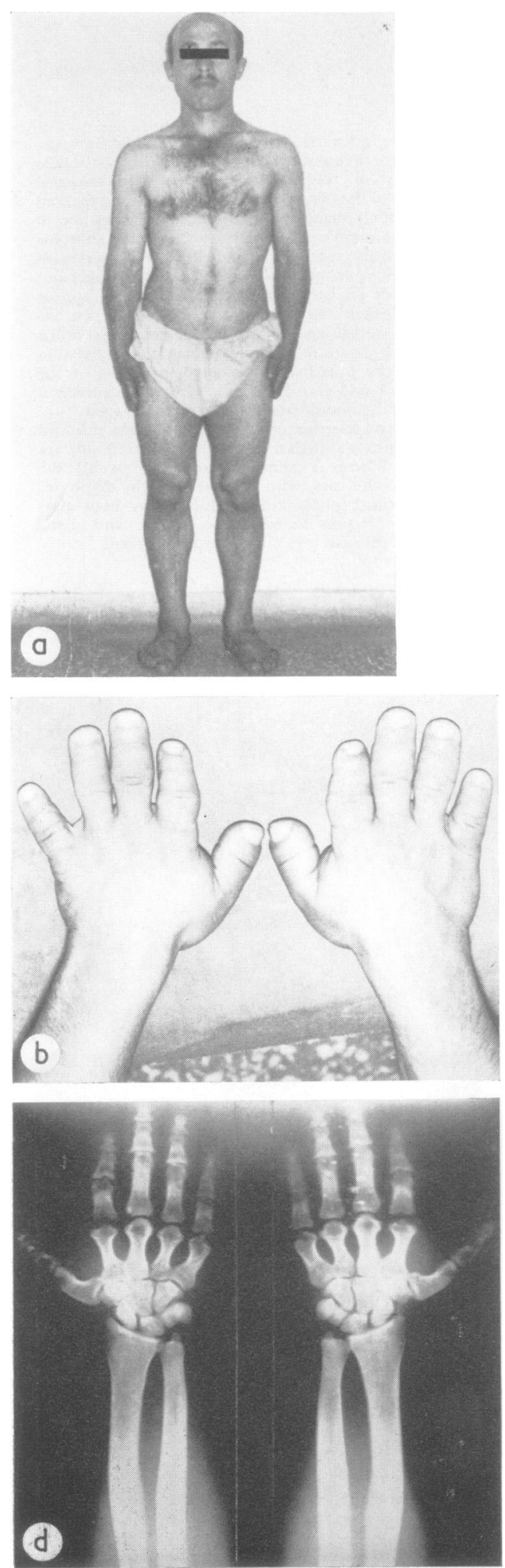

Fig. 3. Case IV.22, a 35-year-old male. Figs. 3a, 3b, and $3 \mathrm{c}$ show the general appearance and hands and feet.

Figs. 3d and 3e show radiographs of the hands and feet of the same patient. Radiological examination of the hand shows symmetrical and marked shortening of all metacarpal bones and phalanges. The proximal phalanges of the third and fourth fingers again make an exception and appear relatively normal. Cone-shaped epiphyses are present on proximal ends of the middle phalanges of the second to fifth fingers.

The toes reveal generalized symmetrical shortening. The second and third metatarsal bones on the left side are relatively normal. The remaining metatarsal bones on this side and all the metatarsal bones of the other foot are short and stubby. Constriction of the diaphyses of the proximal phalanges is again observed.
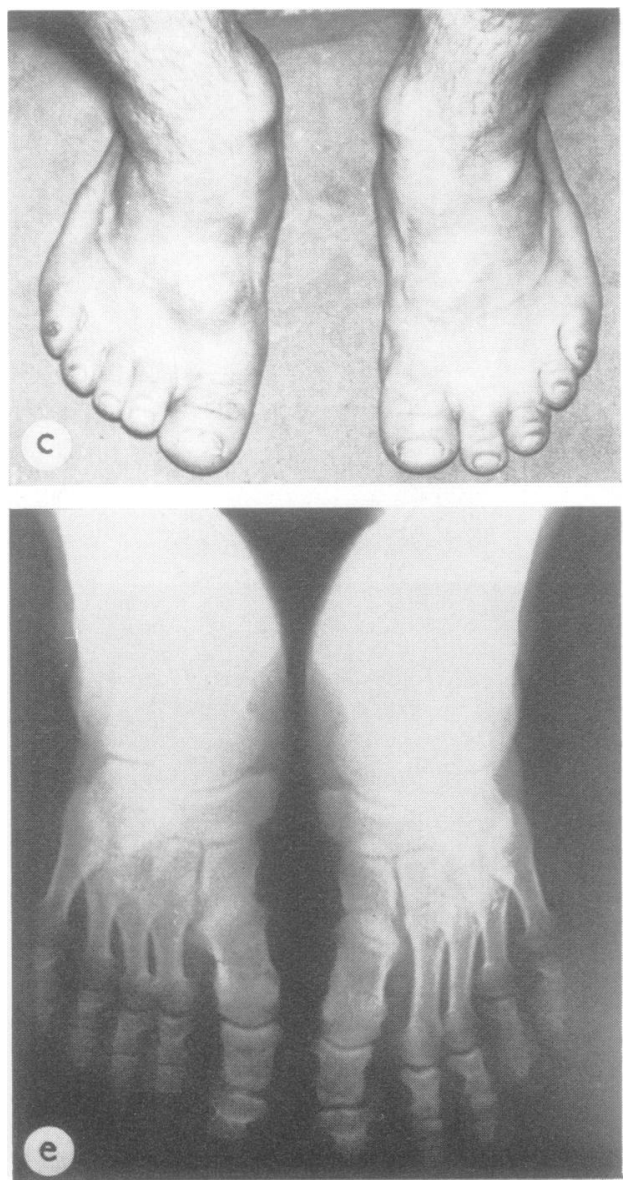
clearance test, intravenous pyelogram, vanyl mandelic acid in 24-hour urine, intravenous regitin test, and renal angiogram. Furthermore, in search of a hypertensive substance, a sample of venous blood was injected into rats.

\section{Results}

Physical examination revealed that the persons who had brachydactyly were uniformly short in stature (males were between 151 and $156 \mathrm{~cm}$, females between 141 and $147 \mathrm{~cm}$ in height) when compared with unaffected family members as well as with other people living in the same area. The patients also had a characteristic general appearance. They had a stocky build and rather round faces. Their hands and feet were broad and square with noticeable shortening of all digits, particularly the index finger, which was markedly shorter than the others (see Figs. 2 and 3). The patients who had brachydactyly also had hypertension. There was no case which had only one of these manifestations. Hypertension seemed to be milder in children, and the level of blood pressure increased progressively with age. The mean values of blood pressure are shown in Table II.

Laboratory studies showed that routine urinalysis, blood counts, blood urea nitrogen, fasting blood sugar, plasma protein, total lipids and cholesterol, serum calcium, phosphorus and alkaline phospha-
TABLE II

MEAN VALUE OF BLOOD PRESSURE IN RELATION TO PATIENT'S AGE

\begin{tabular}{l|c|c}
\hline \multicolumn{1}{c|}{ Age } & Systolic $(\mathrm{mm} / \mathrm{Hg})$ & Diastolic $(\mathrm{mm} / \mathbf{H g})$ \\
\hline Under 10 years & $100-140$ & $60-95$ \\
$10-20$ years & $135-155$ & $75-110$ \\
20-30 years & $160-180$ & $105-115$ \\
Over 30 years & $190-270$ & $110-160$ \\
\hline
\end{tabular}

tase levels, and blood electrolytes were all within normal limits. Skeletal radiology showed no abnormalities except in the hands and feet; $x$-rays of the hands and feet disclosed a large number of anomalies.

1. The carpal bones in all patients appear somewhat shortened in the axial direction, this finding being most pronounced in one patient in whom the width and length of the capitate bone are almost equal.

2. There is shortening of more than one metacarpal and metatarsal bone. This shortening is not always symmetrical, and the number and site of the involved metacarpals and metatarsals vary from patient to patient.

3. All the phalanges of the hands are shortened. However, the proximal and middle phalanges of the

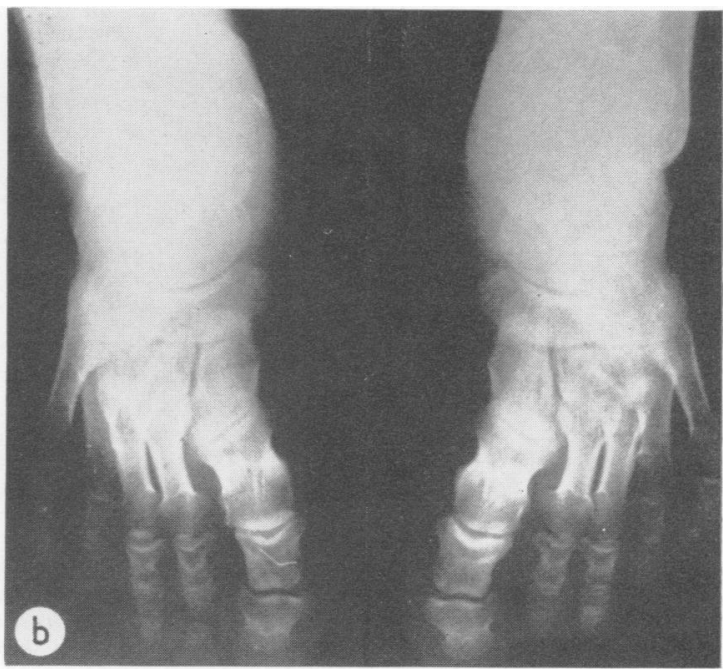

FIGs. 4a and 4b. $X$-rays of the hands and feet of case IV.19, a 42-year-old male. Radiological examination of the hands shows bilateral shortening of all the metacarpals and phalanges with the exception of the proximal and middle phalanges of the third and fourth fingers on both sides which are relatively normal. The middle phalanges of the second fingers reveal cone-shaped epiphyses at their proximal ends. The first, fourth, and fifth metatarsal bones of both feet show shortening with the second and third metatarsals appearing relatively normal. All the phalanges of the toes are shortened and there is constriction of the diaphyses of the proximal phalanges.

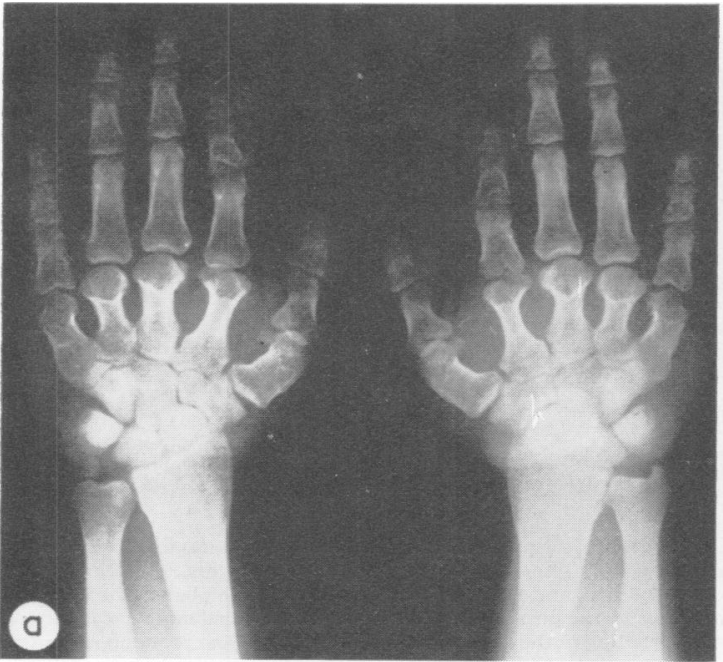





FIGs. 5a and 5b. $X$-rays of the hands and feet of case IV.12, a 36-year-old male. Radiological examination of the hands shows marked shortening of all the metacarpal bones on both sides, with the exception of the proximal and middle phalanges of the third and fourth fingers on both sides, which appeared to be relatively normal. All the phalanges showed similar shortening.

Radiographs of the feet reveal shortening of the first and fourth metatarsals on the right side and the first, third, fourth and fifth metatarsals on the left side. The phalanges of all the toes of both feet are short and display an hour-glass appearance because of constriction of the diaphyses. There is bilateral fusion between the proximal and distal phalanges of the fifth toes.
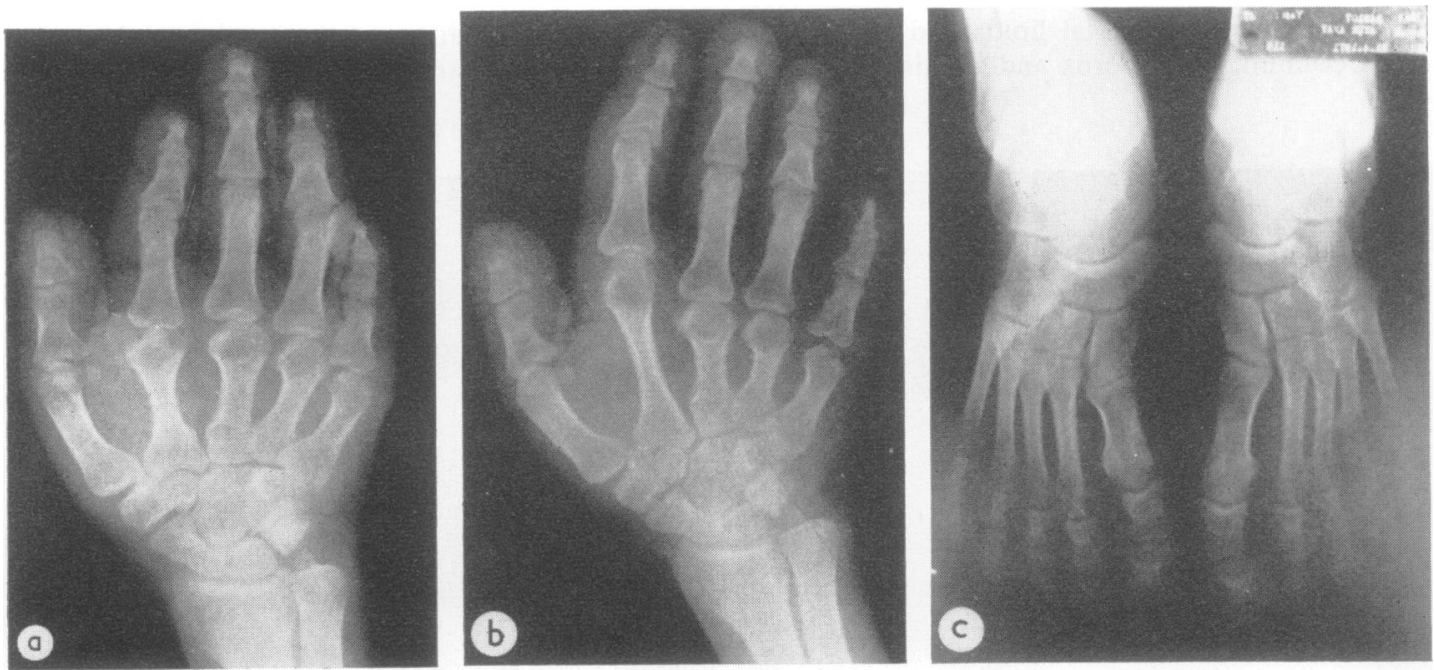

FIGs, 6a, 6b, and 6c. $X$-rays of the left and right hands and the feet of case IV.18, a 46-year-old female. Radiological examination of both hands reveals marked shortening of the second to fifth metacarpals on the right hand. The fingers of both hands show symmetrical shortening of all the phalanges with the exception of the proximal phalanges of the second, third, and fourth fingers and the middle phalanx of the third finger which appeared to be relatively normal. There are cone-shaped epiphyses on the proximal aspects of the middle phalanges of the second and fourth fingers bilaterally. The carpal bones on both sides also display shortening in the axial direction, this being most pronounced in the capitate bones.

The radiograph of the feet of the same patient shows shortening of the first metatarsal on the right and the fourth metatarsal on the left side. The phalanges of all the toes are shortened with fusion of the middle and distal phalanges of the fourth and fifth toes bilaterally. Coneshaped epiphyses are again observed on the proximal phalanges of both first toes. 
third and fourth fingers in all the patients are relatively normal. Some of the involved phalanges reveal cone-shaped epiphyses at their proximal ends. There is universal shortening of all the phalanges of the toes, with a peculiar narrowing of the diaphyses of the proximal phalanges producing an hour-glass appearance. Some of the patients show symmetrical fusion between the middle and distal phalanges of the fourth and fifth toes and others show similar fusion confined only to the fifth toe.

4. The bony architecture and mineralization are quite normal.

Radiology of the hands and feet of three other members of the family are shown in Figs. $4 a$ and $4 b$, $5 \mathrm{a}$ and $5 \mathrm{~b}$, and $6 \mathrm{a}, 6 \mathrm{~b}$, and $6 \mathrm{c}$ (see also Figs. $2 \mathrm{~d}$ and $2 \mathrm{e}$ and $3 \mathrm{~d}$ and $3 \mathrm{e}$ ).

The studies for determining the aetiology of the hypertension were not fruitful. The intravenous pyelograms revealed no abnormalities except in one patient in whom a horse-shoe kidney was found. The creatinine clearance tests, intravenous regitin tests, determination of vanyl mandelic acid, and renal angiograms were within normal limits. The search for hypertensive substance in the venous blood (by injecting into rats) revealed no abnormality. Although renal biopsies were planned for all patients, these could not be done for various reasons. One patient had a horse-shoe kidney, three patients had very high blood pressure which could not be controlled, and another patient refused to grant permission for kidney biopsy.

\section{Discussion}

According to the recent review by McKusick (1971), brachydactyly is classified into 10 groups. In all these groups, the shortness affects either only the phalanges (middle phalanges, middle and terminal phalanges, only thumb and big toes, etc) or only the metacarpal and metatarsal bones. To the best of our knowledge, the presented cases are the first with involvement of the phalanges as well as the metacarpals and metatarsals. Although, in Goeminne's family (1965), the existence of short phalanges and toes by physical examination was mentioned, only two patients out of 15 brachydactylic individuals resembled our patients by radiological examination. In these two patients there was global shortening of the toes in addition to the short metacarpal and metatarsal bones.

Apart from their characteristic brachydactyly, the presence of hypertension in every one of our patients, is probably enough to set them apart as a new entity. It should be mentioned that three patients out of the 15 cases in Goeminne's family also had hypertension. One of them (II.4) developed hypertension at age 70 together with senile cataract; the second one (III.14) had a stomach ulcer at 52 and developed hypertension at age 55, and third one (III.15) had diabetes at age 38 and developed hypertension at age 48 . In these cases, the hypertension seems to be secondary to other diseases and is not related to brachydactyly.

The hypertensive tests gave no clue as to the aetiology of hypertension. In our patients, the pedigree indicates that both brachydactyly and hypertension are genetically transmitted and are most likely to dominant inheritance. It may be that these two conditions are transmitted by a single pleiotropic gene or by two very closely located genes.

\section{REFERENCES}

Bell, J. (1951). On brachydactyly and symphalangism. In Treasury of Human Inheritance, vol. 5, part 1. Cambridge University Press, London and New York.

Farabee, W. C. (1903). Hereditary and Sexual Influence in Meristic Variation. A Study of Digital Malformation in Man. PhD Thesis, Harvard University.

Farabee, W. C. (1905). Inheritance of digital malformation in man. Papers of Peabody Museum, vol. 3, no. 3. Harvard University Press, Cambridge, Mass.

Goeminne, L. (1965). Albright's hereditary poly-osteochondrodystrophy (pseudo-pseudo hypoparathyroidism with diabetes, hypertension, arteritis and polyarthrosis). Acta Geneticae Medicae et Gemellologiae, 14, 226-281.

McKusick, V. A. (1971). Mendelian Inheritance in Man, third edition, pp. 35-37. The Johns Hopkins Press, Baltimore and London. 